\title{
Preliminary studies find DNA erosion in mental disorders
}

The quest for biological markers of mental illness has turned up few good candidates. But emerging evidence suggests that the erosion of telomeres at the ends of chromosomes might be linked to brain function.

Telomeres, the repetitive DNA sequences that cap chromosomes, protect the vulnerable ends of replicating chromosomes in a process similar to how the plastic wrapping at the ends of shoelaces protects them from fraying. In 1990, researchers discovered that telomere length in somatic cells is inversely correlated with age (Nature 346, 866-868; 1990). A newborn child, for example, has between 6,000 and 15,000 repeating telomeric base pairs. But, with each cell replication, the telomeres shorten by a few base pairs. On average, human telomeres disappear at a rate of $40-60$ base pairs per year. This progressively occurs until the telomeres reach a critically short length, impairing the cells from efficiently replicating, eventually leading them to become cancerous, become dormant or die.

Since the 1990s, evidence has accumulated tentatively linking telomere erosion to a suite of clinical disorders. Telomeres seem to erode more rapidly in disorders such as diabetes, obesity, hypertension, cardiovascular disease and chronic stress (Atherosclerosis 195, 83-89; 2007; Obesity, doi:10.1038/oby.2008.413;2008; Intern. Med. 42, 150-153; 2003).

\section{Helpful bacteria harnessed to fight bad bugs}

Doctors and patients must cope with the ever-growing threat of hospital-acquired infections, many of which are caused by 'superbugs' that have evolved resistance to mainstay antibiotics. Researchers are now exploring ways to use 'probiotics', or friendly bacteria, to counteract pathogenic bacteria responsible for these infections.

A new pilot study suggests that probiotics might help prevent ventilatorassociated pneumonia (VAP), one of the most common hospital-acquired infections. VAP occurs when pathogenic microbes, often originating in the mouth or throat, travel down the breathing tube and into the lungs.

Healthcare workers typically swab a patient's mouth with an antiseptic such as chlorhexidine, which can cause allergic reactions, before inserting the tube into the airways. But now a team led by Bengt Klarin of the University Hospital in Lund, Sweden has found evidence that
In 2000, Thomas von Zglinicki's group at Newcastle University's Institute for Ageing and Health in Britain reported that increased telomere erosion is also found in dementia, the first such report in a neurological disorder (Lab. Invest. 80, 1739-1747; 2000). "When I first saw the results, my first impression was that this is nonsense," says von Zglinicki, whose group has since confirmed that telomere length provides a personal history of cell replication, oxidative stress and inflammation, much like the way growth rings divulge information of a tree's chronological age.

But von Zglinicki is still skeptical that information based solely on telomere length can be used to diagnose disorders. "Telomere length depends on many factors, with genetics probably playing the strongest role," says von Zglinicki, who is now conducting research that hopes to use telomere length alongside an arsenal of other measurements (including degree of oxidative stress, DNA damage and inflammation) to form a diagnosis.

In 2003, an analysis of immune cells taken from people with Alzheimer's disease suggested that telomeres erode more rapidly in those with the disorder than their healthy counterparts (Neurobiol. Aging 24, 77-84; 2003).

Some scientists have speculated that

Lactobacillus plantarum 299, a bacterium commonly found in fermented foods, might work just as well as chlorhexidine in preventing harmful bacteria from contaminating breathing tubes. In a randomized controlled trial of 50 ventilated subjects, Klarin and his colleagues found that subjects swabbed with the friendly bacteria solution had no more pathogenic bacteria residing in their upper throats than those swabbed with chlorhexidine (Crit. Care, doi:10.1186/cc7109; 2008).

Klarin's team recently published another study suggesting that individuals given probiotic-containing food alongside their antibiotic treatments might face a lower risk of developing infections with Clostridium difficile, a bacterium that can cause life-threatening gastrointestinal infections (Acta. Anaesthesiol. Scand. 52, 1096-1102; 2008).

Hospitals should be focused on implementing proven, traditional schizophrenia is actually a syndrome of accelerated aging (Schizophr. Bull., doi:10.1093/ schbul/sbm140; 2007). And in February and May of this year, two reports were published hinting that people with schizophrenia have telomere erosion rates almost twice those of normal people (J. Psychiatry Neurosci. 33, 244-247; 2008; Mol. Psychiatry 13, 118 $119 ; 2008)$. "This is significant because it is something that can actually be measured in psychiatric disorders," says Hung-Teh Kao, an author of one of the studies and an associate professor of psychiatry at Brown University.

"Normally, all the diagnostic evidence we gather is from patient interviews, as there is no laboratory test for schizophrenia," says Kao. His study, which looked at the DNA of white blood cells, suggests that, on average, people with schizophrenia in their late 30 s have telomere lengths about 1,700 base pairs shorter than normal people of their age.

Although the exact role of telomere erosion in disease and in the brain remains a mystery, it does provide a glimmer of hope to areas of psychiatry research, such as schizophrenia, where the unsuccessful quest for a pathophysiological explanation has been elusive. "Telomere length may not be the end result," says Kao, "but having something measurable is a first and important step."

David Gruber, New York

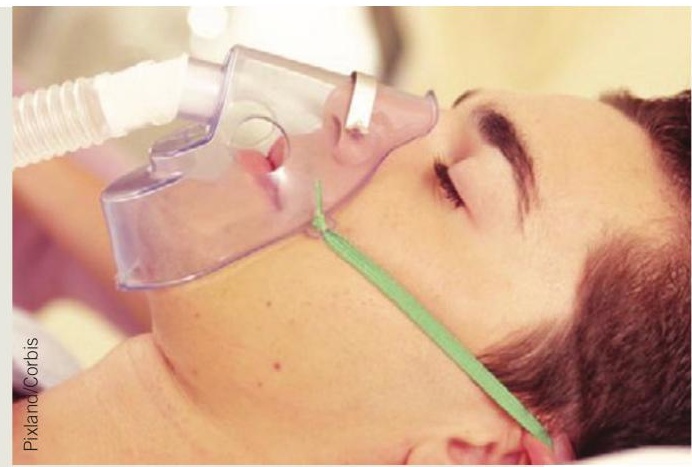

Breathing easy: Bacteria might help

mechanisms of infection control such as environmental cleaning and prudent use of antibiotics, says William Jarvis, a consulting epidemiologist with the Washington, DC-based Association for Professionals in Infection Control and Epidemiology. However, these methods are not foolproof, he acknowledges. "Other interventions such as the use of probiotics need to be evaluated," Jarvis says.

Coco Ballantyne, New York 